\title{
Evaluation of BALB/c mice behavior and relations to the immune response after treatment with $\mathrm{H} 3 \mathrm{~N} 2$ homeopathic solutions
}

\author{
Camila Monteiro Siqueira ${ }^{1}$, Leoni Vilano Bonamin ${ }^{2,3}$, Priscila Dias Motta ${ }^{3}$, \\ Thayná Neves Cardoso ${ }^{3}$, Michelle Sanchez Freitas Correia ${ }^{3}$, \\ José Nelson Couceiro ${ }^{1}$, Carla Holandino ${ }^{1}$
}

(1) Federal University of Rio de Janeiro, Rio de Janeiro, Brazil

(2) University of Santo Amaro, São Paulo, Brazil

(3) Paulista University, São Paulo, Brazil

\begin{abstract}
In Brazil, homeopathic medicines are prepared according to the Homeopathic Pharmacopeia, regulated by ANVISA. Among several categories of medicines, there is the biotherapic group, which is prepared from etiologic agents. In this study, we developed a biotherapic from influenza A virus, aiming the influenza infection prevention. Influenza is a disease that affects thousands of people worldwide every year, with an important economic impact, what motivates the development of new low cost therapies. The H3N2 biotherapic developed in this study was administered to Balb/c mice to evaluate their immune response to viral specific antigens and behavior (homeopathic proving). Sixty-two 4 weeks old Balb/c mice were divided into five experimental groups ( $\mathrm{n}=14$ per group), after approval by the Ethics Committee of Animal Use (Protocol DFBCICB 037) and stimulated daily, blindly, with $1 \%(\mathrm{v} / \mathrm{v})$ different homeopathic medicines, for a maximum period of 42 days. The tested medicines were: biotherapic 30x prepared from inactivated influenza A virus; biotherapic 30x prepared with infectious influenza A virus; and thymulin $5 \mathrm{cH}$, a thymus hormone. The two control groups were treated with water 30x and nothing (baseline group). After 21 days of treatment, half of the animals from each group was challenged subcutaneously with the viral hemagglutinin antigen $(7 \mu \mathrm{g} / 200$ $\mu \mathrm{L}$ ) and monitored by 21 days further, to evaluate the humoral immune response and general behavior, using an open field device. The remaining animals were evaluated by the same behavioral tests at the end of the first 21 days, as an attempt to define the proving features. After euthanasia, all animals were autopsied and the spleen, lungs, heart and mediastine lymph nodes were weighed. Histometry of the spleen follicles was also made. Histopathological and behavioral analyses showed absence of behavioral effects, however, there was increase of spleen lymphoid follicles diameter in immunized animals treated with thymulin and with the biotherapic prepared from infectious influenza A, when compared to the control group. This experiment is being repeated using flow cytometry to complete the analysis and confirm the results.
\end{abstract}

Keywords: Influenza; mice; biotherapic. 


\title{
Avaliação da resposta imune e comportamental de camundongos $\mathrm{BALB} / \mathrm{c}$ após tratamento com soluções homeopáticas de H3N2
}

\begin{abstract}
RESUMO
Medicamentos homeopáticos são preparados de acordo com a farmacotécnica homeopática regulamentada pela ANVISA. Dentre as várias categorias destes medicamentos, destaca-se o grupo dos bioterápicos, medicamentos que são preparados a partir do próprio agente etiológico. No presente estudo, foi desenvolvido um bioterápico a partir do vírus influenza A, visando a profilaxia da gripe. A gripe é uma doença que atinge milhares de pessoas anualmente em todo o mundo e o desenvolvimento de novas terapias para esta doença vem sendo estimulado com frequência. O bioterápico desenvolvido foi administrado a camundongos do tipo Balb/c para avaliação da resposta imune e comportamental. Para tanto, sessenta e dois camundongos Balb/c com 4 semanas de vida foram separados em cinco grupos experimentais, após aprovação pelo Comitê de Ética de Uso de Animais (Protocolo DFBCICB 037) e estimulados diariamente, de maneira cega, por diferentes soluções homeopáticas, na concentração de $1 \%$ (V/V), durante um prazo máximo de 42 dias. Três medicamentos homeopáticos foram testados: bioterápico contendo o vírus influenza A inativado 30DH; bioterápico contendo o vírus influenza A íntegro $30 \mathrm{DH}$; timulina $5 \mathrm{CH}$. Um grupo controle foi tratado com água 30x e o outro não recebeu tratamento. Após 21 dias de tratamento, metade dos animais de cada grupo (31 animais) foi desafiada, por via subcutânea, com o antígeno viral hemaglutinina na concentração de $7 \mu \mathrm{g} /$ $200 \mu \mathrm{L}$ e acompanhados por mais 21 dias para avaliação da resposta imune humoral e do comportamento, pela técnica do campo aberto. Os animais restantes foram submetidos aos mesmos testes ao final dos primeiros 21 dias de tratamento, antes do desafio antigênico. Após a eutanásia, todos os animais foram necropsiados e o baço, o pulmão, o coração e o linfonodo mediastínico foram colhidos para análise de peso e histometria do baço. As análises histopatológica e comportamental mostraram a ausência de efeitos patogenéticos perceptíveis neste modelo experimental, mas houve aumento da reatividade dos folículos linfóides do baço nos animais desafiados antigenicamente e tratados com bioterápico de influenza A íntegro e timulina, em relação ao grupo controle. Este experimento está sendo repetido utilizando citometria de fluxo para ampliar as análises realizadas e confirmar os resultados obtidos.
\end{abstract}

Palavras-chave: Influenza, camundongo, bioterápicos.

\section{(c) ) EY-NC-ND Licensed to GIRI}

Support: CAPES

Conflict of interest: authors declare there is no conflict of interest

Correspondence author: Camila Monteiro Siqueira, camilasiqueira2003@gmail.com .

How to cite this article: Siqueira CM, Bonamin LV, Motta PD, Cardoso TN, Correia MSF, Couceiro JN, Holandino C. Evaluation of BALB/c mice behavior and relations to the immune response after treatment with H3N2 homeopathic solutions. Int J High Dilution Res [online]. 2011 [cited YYYY Month dd]; 10(36): 128-129. Proceedings of the XXV GIRI Symposium and VIII CBFH; 2011 Sep 04-07; Foz do Iguaçu (Brazil). GIRI and ABFH; 2011; Available from: http://www.feg.unesp.br/ ojs/index.php/ijhdr/article/view/474/489 